\title{
A NOTE ON COCYCLES OF UNITARY REPRESENTATIONS
}

\author{
W. PARRY AND K. SCHMIDT
}

\begin{abstract}
Given a unitary representation $U$ of a locally compact abelian group $G$, we investigate the relationship between two cocycles $a_{1}, a_{2}$ : $V a_{1}=a_{2}+b$ for some unitary operator $V$ commuting with $U$ and some coboundary $b$. A necessary and sufficient condition is given in terms of canonical-finite measures defined on $G-1$. These results are applied to the representation of $Z$ defined by the shift of a stationary Markov chain.
\end{abstract}

1. Introduction. Let $G$ be a locally compact second countable abelian group and let $U$ be a continuous unitary representation of $G$ in a complex separable Hilbert space $H$ which does not contain the trivial representation. A cocycle of $U$ is a continuous map $a: G \rightarrow H$ satisfying $U_{g_{1}} a\left(g_{2}\right)-a\left(g_{1}+g_{2}\right)+$ $a\left(g_{1}\right)=0$ for all $g_{1}, g_{2}$ in $G$. If $a(g)=U_{g} v-v$ for some $v$ in $H$ and all $g$ in $G, a$ is called a coboundary. Two cocycles are called cohomologous if their difference is a coboundary. We shall, in fact, be interested in a more general relation between cocycles: Given two cocycles $a_{1}$ and $a_{2}$ for $U$, when does there exist a unitary operator $V$ on $H$ such that $V a_{1}$ and $a_{2}$ are cohomologous cocycles for $U$ ? This problem arose in [1] for the special case of $G=\mathbf{Z}$. There a numerical invariant (variance) of this relation was introduced. The connection between the variance and the results presented here are discussed at the end of this paper. Our first object is to present a necessary and sufficient condition for a cocycle to be a coboundary. Apparently this theorem is known to a number of mathematicians, but as far as we are aware, no proof has been published before. ${ }^{1}$

2. Coboundaries. In this section the topology and specific nature of the group (or semigroup) $G$ is irrelevant.

THEOREM 2.1. Let $U$ be a unitary representation of the group $G$ in a complex separable Hilbert space $H$. A cocycle a of $U$ is a coboundary if and only if $a$ is bounded, i.e. if $\sup _{g \in G}\|a(g)\|<\infty$.

Proof. Clearly every coboundary $a$ for $U$ is bounded. On the other hand, if $a$ is a bounded cocycle, we define $T_{g}: H \rightarrow H$ by $T_{g} v=a(g)+U_{g} v$ for every $g \in G, v \in H$. Each $T_{g}$ is affine and

$$
T_{g_{1}} T_{g_{2}}(v)=T_{g_{1}}\left(a\left(g_{2}\right)+U_{g_{2}} v\right)=a\left(g_{1} g_{2}\right)+U_{g_{1} g_{2}} v=T_{g_{1} g_{2}} v,
$$

Received by the editors June 12, 1974.

AMS (MOS) subject classifications (1970). Primary 22D10.

Key words and phrases. Unitary representations, cocycles, coboundaries.

${ }^{1}$ Added in proof. We are mistaken. A proof appears in The mean ergodic theorem and saturation, Indiana Math. J. $20(1970 / 71), 1163-1174$, by P. L. Butzer and U. Westphal. 
so that $T$ is an affine representation of $G$. Since $a$ is bounded, the (weak) closure $K$ of the convex hull of $\{a(g), g \in G\}$ is weakly compact and invariant under each $T_{g}$. Moreover, $T_{g}$ acts distally (in fact, isometrically) on $K$ with respect to the norm topology. Hence, by the Ryll-Nardzewski fixed point theorem [2], there exists a $v \in K$ such that $T_{g} v=v$ and, consequently, $a(g)=v-U_{g} v$ for all $g \in G$.

REMARK 2.2. Theorem 2.1 has an obvious generalisation to bounded representations on reflexive Banach spaces.

3. The main theorem. Let $G$ be a locally compact second countable abelian group and let $U$ be a continuous unitary representation of $G$ in a complex separable Hilbert space $H$ which does not contain the trivial representation. If $\hat{G}$ denotes the character group of $G$, and $U_{g}=\int_{\hat{G}} \chi(g) d P(\chi)$ is the spectral form of $U$, where $P$ is a projection valued measure on $\hat{G}$, then our assumption means that $P\{1\}=0$ ( 1 is the identity of $\hat{G}$ ). Let $a$ be a continuous cocycle of $U$. We denote by $H_{a}$ the subspace of $H$ spanned by $\{a(g), g \in G\}$. The following lemma is contained in [3, pp. 96-97]:

LeMmA 3.1. There exists a unique o-finite measure $F_{a}$ on $\hat{G}-\{1\}$ and a unitary map $W_{a}: H_{a} \rightarrow L_{2}\left(\hat{G}-\{1\}, F_{a}\right)$ such that $W_{a} a(g)(\chi)=\chi(g)-1$ for all $g \in G, \chi \in \hat{G}$.

LEMMA 3.2. Let $a_{1}$ and $a_{2}$ be two cocycles of $U$, and let $F_{a_{i}}, i=1,2$, be the corresponding measures on $\hat{G}-\{1\}$ defined in Lemma 3.1. The following conditions are equivalent:

(1) $F_{a_{1}}=F_{a_{2}}$.

(2) There exists a unitary operator $V$ on $H$ which commutes with $U$ and which satisfies $V a_{1}=a_{2}$.

Proof. Define $H_{a_{i}}, i=1,2$, as before and denote by $U_{g}^{(i)}$ the restriction of $U_{g}$ to the invariant subspaces $H_{a_{i}}, i=1,2$. We write $U_{g}^{(i)}=\int_{\hat{G}} \chi(g) d P^{(i)}(\chi)$ for the spectral representation of $U^{(i)}$. As an immediate consequence of Lemma 3.1, we conclude that the multiplicity of $P^{(i)}$ is everywhere $\leqslant 1$. Assume now that $F_{a_{1}}=F_{a_{2}}$. The map $T: H_{a_{1}} \rightarrow H_{a_{2}}$ given by $T=W_{a_{2}}^{-1} W_{a_{1}}$ is unitary. It is easy to see that $T$ can be extended to a unitary operator on the closed joint linear span of $H_{a_{1}}$ and $H_{a_{2}}$, and finally to a unitary operator $V$ on $H$ which commutes with $U$. Then $V$ satisfies the conditions in (2). The converse is obvious from Lemma 3.1 .

LEMMA 3.3. $a$ is a coboundary if and only if $F_{a}$ is totally finite.

Proof. If $F_{a}$ is totally finite, $a$ is bounded and hence, by Theorem 1.1, a coboundary. Conversely, if $a$ is bounded, then $a(g)=U_{g} v-v$ where $v \in H_{a}$ and where $-v$ is a fixed point of the map $y \rightarrow U_{g} y+a(g)$ from $H_{a}$ to $H_{a}$, for every $g \in G$. So $W_{a} v$ must be the function 1 in $L_{2}\left(\hat{G}-\{1\}, F_{a}\right)$, which implies that $F_{a}$ is totally finite.

We can now state the main result:

THEOREM 3.4. Let $G$ be a locally compact second countable abelian group, and let $U$ be a continuous unitary representation of $G$ in a complex separable Hilbert space $H$. Assume that $U$ does not contain the trivial representation, and consider two cocycles $a_{1}$ and $a_{2}$ of $U$. We define the corresponding measures $F_{a_{1}}$ 
and $F_{a_{2}}$ on $\hat{G}-\{1\}$ as in Lemma 3.1. Then the following statements are equivalent:

(1) There exists a unitary operator $V$ on $H$ such that

$$
V a_{1}(g)=a_{2}(g)+b(g), \quad g \in G,
$$

where $b$ is a coboundary for $U$.

(2) For any $\sigma$-finite measure $\rho$ on $\hat{G}-\{1\}$ with $F_{a_{i}} \ll \rho, i=1,2$, we have

$$
\delta\left(a_{1}, a_{2}\right)=\int\left|\left(\frac{d F_{a_{1}}}{d \rho}\right)^{1 / 2}-\left(\frac{d F_{a_{2}}}{d \rho}\right)^{1 / 2}\right|^{2} d \rho<\infty .
$$

Before we prove this theorem, two remarks are in order.

REMARKS 3.5. (1) The operator $V$ can always be chosen to commute with the representation $U$.

(2) $\delta\left(a_{1}, a_{2}\right)$ is independent of the measure $\rho$ chosen as long as $\rho$ dominates both $F_{a_{i}}, i=1,2$.

Proof of Theorem 3.4. Assume (1) holds. Let $\tilde{H}$ denote the closed subspace of $H$ spanned by $\left\{V a_{1}(g), a_{2}(g), g \in G\right\}$. Modifying $V$ if necessary, we may assume that the spectral measure $\tilde{P}$ in the spectral representation $\tilde{U}=\int_{\hat{G}} \chi d \tilde{P}(\chi)$ of the restriction $\tilde{U}$ of $U$ to $\tilde{H}$ has multiplicity $\leqslant 1$ everywhere. Applying well-known results we can find a unitary map $\tilde{W}$ from $\tilde{H}$ to $L_{2}(\hat{G}-\{1\}, \rho)$, where $\rho$ is some $\sigma$-finite measure on $\hat{G}-\{1\}$, such that $W U_{g} W^{-1}$ is multiplication by the function $\chi \rightarrow \chi(g)$ in $L_{2}(\hat{G}-\{1\}, \rho)$. It follows again from [3, pp. 96-97] that there exist two complex valued functions $f_{1}$ and $f_{2}$ on $\hat{G}-\{1\}$, such that $W V a_{1}(g)(\chi)=f_{1}(\chi) \cdot(\chi(g)-1)$ and $W a_{2}(g)(\chi)=f_{2}(\chi) \cdot(\chi(g)-1)$ for all $g \in G$ a.e. $(\chi)$. Since $V a_{1}$ and $a_{2}$ differ only by a coboundary, the same is true for $W V a_{1}$ and $W a_{2}$, so that $\left(f_{1}(\chi)-f_{2}(\chi)\right) \cdot(\chi(\cdot)-1)$ is a coboundary. A straightforward application of Lemmas 3.1 and 3.3 shows that the measure $F$ given by

$$
d F(\chi)=\left|f_{1}(\chi)-f_{2}(\chi)\right|^{2} d \rho(\chi)
$$

is totally finite, so that $f_{1}-f_{2} \in L_{2}(\hat{G}-\{1\}, \rho)$. But we also have $d F_{a_{i}}(\chi)=\left|f_{i}(\chi)\right|^{2} d \rho(\chi)$ from the uniqueness of $F_{a_{i}}$ in Lemma 3.1, which gives

$$
\int\left|\left(\frac{d F_{a_{1}}}{d \rho}\right)^{1 / 2}-\left(\frac{d F_{a_{2}}}{d \rho}\right)^{1 / 2}\right|^{2} d \rho=\int\left(\left|f_{1}\right|-\left|f_{2}\right|\right)^{2} d \rho \leqslant \int\left|f_{1}-f_{2}\right|^{2} d \rho<\infty
$$

and thus proves (2). Conversely, if (2) holds, put $\rho=F_{a_{1}}+F_{a_{2}}$ and consider the linear isometries $\tilde{V}_{a_{i}}: H_{a_{i}} \rightarrow L_{2}(\hat{G}-\{1\}, \rho)$ defined by

$$
\left(\tilde{V}_{i} a_{i}(g)\right)(\chi)=\left(d F_{a_{i}}(\chi) / d \rho\right)^{1 / 2}(\chi(g)-1) .
$$

By assumption (2), $\tilde{V}_{1} a_{1}(g)=\tilde{V}_{2} a_{2}(g)+\tilde{b}(g), g \in G$, where $\tilde{b}_{\tilde{U}}$ is a coboundary for the representation $\tilde{U}$ of $G$ in $L_{2}(\tilde{G}-\{1\}, \rho)$ given by $\left(\tilde{U}_{g} f\right)(\chi)$ $=\chi(g) f(\chi)$. We now define an isometry $T: L_{2}(\hat{G}-\{1\}, \rho) \rightarrow H$ such that $T \tilde{V}_{2}$ is the identity on $H_{a_{2}}$, and $T \tilde{U}_{g} T^{-1}$ is the restriction of $U$ onto $T\left(L_{2}(\hat{G}-\{1\}, \rho)\right)$. (That such a $T$ exists is straightforward.) $T \tilde{V}_{1}$ is an 
isometry of $H_{a_{1}}$ which can be extended to a unitary operator $V$ on $H$ which commutes with $U$, and which satisfies $V a_{1}=a_{2}+b$ where $b$ is a coboundary. The proof is complete.

COROllary 3.6. If $G=\mathbf{Z}$ (the group of integers), and if $a_{1}$ and $a_{2}$ are two cocycles of a unitary representation $U$ of $\mathbf{Z}$ in $H$ which does not contain the trivial representation, we can write $\left(U_{n}=U^{n}\right)$

$$
a_{i}(n)=u_{i}+U u_{i}+\cdots+U^{n-1} u_{i}
$$

for some vectors $u_{i} \in H, i=1,2$, and all positive integers $n$. Let $\tilde{u}_{i}$ stand for the spectral measures of $u_{i}$ on $\hat{\mathbf{Z}}=K=\{z:|z|=1\}$, i.e. $\left\langle U^{n} u_{i}, u_{i}\right\rangle=\int_{K} \lambda^{n} d \tilde{u}_{i}$ for $i=1,2$ and all $n \in \mathbf{Z}$. Then we obtain $d F_{a_{i}}(\lambda)=d u_{i}(\lambda) /|1-\lambda|^{2}$.

Theorem 3.4 reads now as follows: There exists a unitary operator $V$ on $H$ (commuting with $U$ ) and an element $w$ in $H$ such that $u_{2}=V u_{1}+U w-w$, if and only if

$$
\int_{K}\left(\left(\frac{d \tilde{u}_{1}}{d \rho}\right)^{1 / 2}-\left(\frac{d \tilde{u}_{2}}{d \rho}\right)^{1 / 2}\right)^{2} \frac{d \rho}{|1-\lambda|^{2}}<\infty
$$

for some (and therefore all) measures $\rho \gg \tilde{u}_{1}, \tilde{u}_{2}$ on $K$.

4. The special case $G=\mathbf{Z}$. Let $H$ be a complex separable Hilbert space, and let $U$ be a unitary operator on $H$ which does not have 1 in its discrete spectrum. Consider the representation $n \rightarrow U^{n}$ of $\mathbf{Z}$ in $H$, and let $u \in H$. In [1] the numerical invariant variance $\sigma^{2}(U, u)$ was introduced:

$$
\begin{aligned}
\sigma^{2}(U, u) & =\lim _{N \rightarrow \infty} \frac{1}{N}\left\|u+\cdots+U^{N} u\right\|^{2} \\
& =\|u\|^{2}+2 \operatorname{Re} \lim _{N \rightarrow \infty} \frac{1}{N} \sum_{j=1}^{N}(N-j)\left\langle U^{j} u, u\right\rangle
\end{aligned}
$$

when these limits exist ( $\operatorname{Re}$ denotes real part). If $\sum_{n=1}^{\infty}\left\langle U^{n} u, u\right\rangle$ converges, then $\sigma^{2}(U, u)=\|u\|^{2}+2 \operatorname{Re} \sum_{j=1}^{\infty}\left\langle U^{j} u, u\right\rangle$ is a numerical invariant of the relationship $u_{1}=V u_{2}+U w-w$. In other words,

Proposition 4.1 [1]. If $U$ is a unitary operator on $H$ with no nonzero fixed vector, $V$ a unitary operator on $H$ which commutes with $U$, and $u_{1}, u_{2}$ and $w$ are elements of $H$, then the relation $u_{1}=V u_{2}+U w-w$ implies $\sigma^{2}\left(U, u_{1}\right)$ $=\sigma^{2}\left(U, u_{2}\right)$.

We wish to examine the extent to which the converse of this proposition holds when $u_{1}, u_{2}$ have continuous densities $f_{i}$ with respect to the Lebesgue measure $d \lambda$ on the circle $K$.

THEOREM 4.2. If $U$ is a unitary operator on $H$ with no nonzero fixed vector, and if $u_{1}, u_{2} \in H$ have spectral measures $\tilde{u}_{1}, \tilde{u}_{2}$ with continuous densities $f_{1}, f_{2}$ on $K$ such that $f_{i}^{1 / 2}$ is differentiable at 1 for $i=1,2$, then $\sigma^{2}\left(U, u_{1}\right)=\sigma^{2}\left(U, u_{2}\right)$ implies that there exist a unitary operator $V$ commuting with $U$, and $a w \in H$ so that $u_{2}=V u_{1}+U w-w$.

Proof. We first note that 


$$
\begin{aligned}
\sigma^{2}\left(U, u_{i}\right)= & \lim _{N \rightarrow \infty} \frac{1}{N}\left\|u_{i}+\cdots+U^{N} u_{i}\right\|^{2} \\
= & \lim _{N \rightarrow \infty} \frac{1}{N} \int\left|1+\cdots+\lambda^{N}\right|^{2} d \tilde{u}_{i}(\lambda) \\
& \times \lim _{N \rightarrow \infty} \frac{1}{N} \int\left|\frac{\lambda^{N+1}-1}{\lambda-1}\right|^{2} f_{i}(\lambda) d \lambda=f_{i}(1)
\end{aligned}
$$

by a well-known theorem of harmonic analysis. Hence

$$
\begin{gathered}
\int_{K}\left(\left(\frac{d \tilde{u}_{1}}{d \lambda}\right)^{1 / 2}-\left(\frac{d \tilde{u}_{2}}{d \lambda}\right)^{1 / 2}\right)^{2} \frac{d \lambda}{|1-\lambda|^{2}}=\int_{K}\left(\frac{f_{1}(\lambda)^{1 / 2}-f_{2}(\lambda)^{1 / 2}}{|1-\lambda|}\right)^{2} d \lambda \\
=\int_{K}\left(\frac{f_{1}(\lambda)^{1 / 2}-f_{1}(1)^{1 / 2}}{|1-\lambda|}+\frac{f_{2}(1)^{1 / 2}-f_{2}(\lambda)^{1 / 2}}{|1-\lambda|}\right)^{2} d \lambda .
\end{gathered}
$$

Differentiability of $f_{i}^{1 / 2}$ at 1 ensures that $\left(f_{i}(\lambda)^{1 / 2}-f_{i}(1)^{1 / 2}\right) /|1-\lambda|$ is bounded on $K$ for $i=1,2$. Consequently

$$
\int_{K}\left(\left(\frac{d \tilde{u}_{1}}{d \lambda}\right)^{1 / 2}-\left(\frac{d \tilde{u}_{2}}{d \lambda}\right)^{1 / 2}\right)^{2} d \lambda<\infty,
$$

and Corollary 3.6 completes the proof.

5. Representations induced by Markov chains. Let $P$ be an irreducible, aperiodic, stochastic $k \times k$ matrix and let $\Lambda P=\Lambda$ be the left $P$ invariant positive vector representing stationary initial probabilities of the Markov chain. It is well known how a stationary probability $m$ is derived from $\Lambda$ and $P$, so that the shift transformation $T$ on the space $X$ of sequences of $k$ symbols (states) preserves the measure $m$. Let $U$ be the unitary operator on $H=L_{2}(X, m)$ given by $(U f)(x)=f(T x)$. The number $\sigma^{2}(U, u)=\sigma^{2}(T, u)$ was introduced for special vectors $u$ arising in stochastic processes, namely the centralized information function. In the case of Markov chains this function $u$ is a function of two states, i.e. $u(x)=u\left(x_{0}, x_{1}\right)$ for any $x=(\cdots$, $\left.x_{-1}, x_{0}, x_{1}, \cdots\right)$ in $X$. We devote this section to the problem of finding $\sigma^{2}(T, u)$ and $\tilde{u}$ when $u$ is a function of two states such that $\int u d m=0$.

Lemma 5.1. For every $y={ }^{t}\left(y_{1} \cdots y_{k}\right) \in \mathbf{C}^{k}$ ( ${ }^{t}$ denotes transpose), we have

$$
P^{n}\left(y-\left(\sum_{i=1}^{k} \lambda_{i} y_{i}\right) 1\right) \rightarrow 0
$$

where $\Lambda=\left(\lambda_{1}, \ldots, \lambda_{k}\right)$ and $1={ }^{t}(1 \cdots 1)$.

Proof. 1 is the unique column vector corresponding to the eigenvalue 1 , and all other eigenvalues have modulus less than 1 by the Perron-Frobenius theorem. Hence $\mathbf{C}^{k}=V_{1} \oplus V_{C}$ where $V_{C}=\left\{\zeta \in \mathbf{C}^{k}: P^{n} \zeta \rightarrow 0\right\}$, and $V_{1}$ $=\{\mu 1, \mu \in \mathbf{C}\}$. Consequently we have, for any $y \in \mathbf{C}^{k}$ with $y=\mu 1+\zeta$ for some $\mu \in \mathbf{C}, \zeta \in V_{C}, P^{n} y=\mu 1+P^{n} \zeta \rightarrow \mu 1$. On the other hand, $P^{n}$ $\rightarrow^{t}(\Lambda \cdots \Lambda)$ so that $\sum_{i=1}^{k} \lambda_{i} y_{i}=\mu$. This completes the proof. 
THEOREM 5.2. Let $u(x)=u\left(x_{0}, x_{1}\right)$ be a function of two states on $X$ with $\int u d m=0$. Then $u$ has a $C^{\infty}$-spectral density $f(i . e . d \tilde{u}(\lambda)=f(\lambda) d \lambda$, where $f$ is $\left.C^{\infty}\right)$, and

$$
\sigma^{2}(T, u)=f(1)=\int|u|^{2} d m+2 \operatorname{Re} \sum_{i, j=1}^{k} \lambda_{i} p_{i, j} \bar{c}_{j} u(i, j)
$$

where $c \in V_{C}$ satisfies

$$
(I-P) c=b, \quad b_{i}=\sum_{j} p_{i, j} u(i, j) .
$$

Proof. For any $n$ we have

$$
\begin{aligned}
\left\langle U^{n} u, u\right\rangle & =\int f T^{n} \bar{f} d m=\sum u(i, j) \overline{u(k, l)} \lambda_{i} p_{i, j} p_{j, k}^{n-1} p_{k, l} \\
& =\left\langle a, P^{n-1} b\right\rangle,
\end{aligned}
$$

where $a_{j}=\sum_{i} u(i, j) \lambda_{j} p_{i, j}, b_{k}=\sum_{1} u(k, l) p_{k, l}$. Note that $\sum_{j} a_{j}=0$, since $\int u d m$ $=0$. Evidently, $\Sigma_{k} \lambda_{k} b_{k}=\Sigma_{k, l} \lambda_{k} u(k, l) p_{k, l}=0$, so that $b \in V_{C}$. Since for any element $y \in V_{C}, P^{n} y$ tends to zero exponentially, $\left\langle U^{n} u, u\right\rangle \rightarrow 0$ exponentially as $|n| \rightarrow \infty$. It is well known that this implies $\left\langle U^{n} u, u\right\rangle=\int \lambda^{n} f(\lambda) d \lambda$ where $f$ is $C^{\infty}$. Hence

$$
\begin{aligned}
f(\lambda) & =2 \operatorname{Re} \sum_{n=1}^{\infty} \lambda^{-n}\left\langle a_{1} P^{n-1} b\right\rangle+\int|u|^{2} d m \\
& =\int|u|^{2} d m+2 \operatorname{Re} \lambda^{-1} \sum_{n=1}^{\infty}\left\langle a_{1}(\lambda P)^{n-1} b\right\rangle \\
& =\int|u|^{2} d m+2 \operatorname{Re} \lambda^{-1}\left\langle a,(I-\lambda P)^{-1} b\right\rangle
\end{aligned}
$$

which makes sense on $V_{C}$, since $\lambda P$ is nilpotent there. Substituting $\lambda=1$ we have proved the theorem.

\section{REFERENCES}

1. R. Fellgett and W. Parry, Endomorphisms of a Lebesgue space. II, Bull. London Math. Soc. 7 (1975), 151-158.

2. F. P. Greenleaf, Invariant means on topological groups and their applications, Van Nostrand Math. Studies, no. 16, Van Nostrand Reinhold, New York, 1969. MR 40 \#476.

3. K. R. Parthasarathy and K. Schmidt, Positive definite kernels, continuous tensor products, and central limit theorems of probability theory, Lecture Notes in Math., vol. 272, Springer-Verlag, Berlin and New York, 1972.

Mathematics Institute, University of Warwick, Coventry, England 